\title{
Phase Shift of Interacting Algebraic Solitary Waves in a Two-Layer Fluid System
}

\author{
Y. Matsuno \\ Department of Physics, Faculty of Liberal Arts, Yamaguchi University, Yamaguchi 753, Japan
}

(Received 28 March 1995)

\begin{abstract}
The interaction of interfacial solitary waves of algebraic type is investigated on the basis of a higherorder Benjamin-Ono equation. By developing a multisoliton perturbation theory, we show analytically that the overtaking collision between two solitary waves exhibits the phase shift but the amplitudes are not altered after interaction. The predication of the phase shift that takes place between algebraic solitary waves is the first example reported in the literature.
\end{abstract}

PACS numbers: 03.40.Kf, 02.90.+p, 03.40.Gc, 68.10.-m

The development of the theory of nonlinear waves has enabled us to describe the wave propagation in physical systems by simple nonlinear evolution equations (NEEs) [1-3]. A typical example is the Korteweg-de Vries $(\mathrm{KdV})$ equation that is a model for the unidirectional propagation of long waves of small amplitude. Almost all the NEEs thus derived incorporate the lowest-order nonlinearity in wave amplitude. For the study of large amplitude waves, however, one must take into account higher-order nonlinearities. In the context of water waves, various types of higher-order $\mathrm{KdV}$ equations have been derived in accordance with the physical situation under consideration and the properties of solutions have been studied in detail. For instance, the interaction process between two solitary waves has been investigated both analytically [4-6] and numerically [7-10].

Recently, the author derived a higher-order BenjaminOno (BO) equation that describes interfacial waves in a two-layer fluid system and obtained a solitary wave solution [11]. In contrast to the great deal of work which has been done for the higher-order $\mathrm{KdV}$ equations, there appears to be a dearth of study dealing with the effect of higher-order nonlinear and dispersive terms on the $\mathrm{BO}$ equation. The BO solitons have novel characteristics [12$14]$ in comparison with those of the $\mathrm{KdV}$ equation. One is that they have a profile expressed in terms of algebraic functions. Another remarkable aspect is that they exhibit no phase shift after collisions. Our primary concern is to study how these important characteristics are altered owing to the addition of higher-order terms.

In this Letter, we shall focus our attention on the interaction process of solitary waves. In particular, the total changes of the amplitude and the phase after overtaking collision between two solitary waves are calculated analytically by developing a multisoliton perturbation theory for the BO equation. It will be shown that the amplitudes of solitary waves do not change after interaction while the phase shifts occur depending on the amplitude ratio of both solitary waves and the density ratio of two layers.

The basic equation considered here is a higher-order BO equation derived in a recent paper [11] to describe a unidirectional motion of interfacial waves in a two-layer fluid system in which the upper layer with a uniform density $\rho_{2}$ is infinitely deep and the depth of the lower layer with a uniform density $\rho_{1}$ is very small compared with the typical wavelength of the wave. It can be written in dimensionless form as follows [1]:

$$
\begin{aligned}
& \eta_{t}+\eta_{x}+\frac{3}{2} \alpha \eta \eta_{x}+\frac{1}{2} \Delta \delta H \eta_{x x}-\frac{3}{8} \alpha^{2} \eta^{2} \eta_{x} \\
&+\frac{1}{2} \Delta \alpha \delta\left[\frac{5}{4} \eta H \eta_{x x}\right.\left.+\frac{9}{4} H\left(\eta \eta_{x}\right)_{x}+\eta_{x} H \eta_{x}\right] \\
&-\frac{3}{8}\left(\Delta^{2}-\frac{4}{9}\right) \delta^{2} \eta_{x x x}=0 .
\end{aligned}
$$

Here $\eta=\eta(x, t)$ is the interfacial elevation, $\Delta=\rho_{2} /$ $\rho_{1}(<1)$ is the density ratio, $\alpha$ and $\delta$ are small parameters given by $\alpha=a / h_{0}, \delta=h_{0} / l$ where $a$ and $l$ are typical amplitude and wavelength of the wave, respectively, and $h_{0}$ is the undisturbed depth of the lower layer. The operator $H$ is the Hilbert transform and the subscripts $t$ and $x$ appended to $\eta$ denote partial differentiations. It should be remarked that in the derivation of (1) the ordering $\alpha=O(\delta)$ has been introduced between the two parameters. In the first order of the approximation, Eq. (1) reduces to the $\mathrm{BO}$ equation provided that the condition $\alpha \ll \Delta<1$ holds, which is assumed implicitly throughout the analysis. Equation (1) can be recast in a form relevant to the following perturbation analysis as

$$
\begin{aligned}
u_{t}+4 u u_{x}+H u_{x x}= & \epsilon\left[3 u^{2} u_{x}-\frac{15}{4} u H u_{x x}-\frac{27}{4} H\left(u u_{x}\right)_{x}\right. \\
& \left.-3 u_{x} H u_{x}+\frac{27}{16 \Delta^{2}}\left(\Delta^{2}-\frac{4}{9}\right) u_{x x x}\right] \\
\equiv & \epsilon R[u]
\end{aligned}
$$

by transforming it to a moving frame with the phase velocity of the wave and then rescaling the variables $\eta, t, x$ according to $\eta \rightarrow(3 \epsilon / \alpha) u, t \rightarrow\left(32 \Delta \delta / 81 \epsilon^{2}\right) t$, and $x \rightarrow$ $(4 \Delta \delta / 9 \epsilon) x$, respectively, where $\epsilon$ is a small positive parameter and $\epsilon R[u]$ represents a perturbation.

In order to analyze soliton equations with small perturbations, several different approaches are known. These include a method based on the inverse scattering transform [15], a direct method using multiple time scale expansion 
[16], a mixture of the above two methods [17], a technique using variational principle [18], and a generalized reductive perturbation method $[4,5,19]$. As for the merits and demerits of these methods, see a review paper [20]. It should be pointed out, however, that for the perturbed BO equation, these methods have not been applied as yet. Quite recently, a direct multisoliton perturbation theory has been developed that is applicable to a wide class of NEEs [21]. Therefore, we first summarize the main results of the theory with an application to the present problem.

To begin with, expand $u$ in powers of $\epsilon$ as $u=$ $\sum_{j=0}^{\infty} \epsilon^{j} u_{j}$ and introduce the multiple times by $t_{j}=$ $\epsilon^{j} t(j=0,1, \ldots)$, so that the time derivative is replaced by $\partial / \partial t=\sum_{j=0}^{\infty} \epsilon^{j} \partial / \partial t_{j}$, where $u_{j}$ are supposed to be functions of $t_{0}, t_{1}, \ldots$ as well as $x$. Substituting these expressions into (2) and equating the coefficients of like powers of $\epsilon$, we obtain a hierarchy of equations for $u_{j}(j=0,1, \ldots)$, the first two members of which read in the forms

$$
\begin{gathered}
u_{0, t_{0}}+4 u_{0} u_{0, x}+H u_{0, x x}=0, \\
u_{1, t_{0}}+4\left(u_{0} u_{1}\right)_{x}+H u_{1, x x}=R\left[u_{0}\right]-u_{0, t_{1}} .
\end{gathered}
$$

Equation (3) is just the BO equation and the equations for $j \geq 1$ become linear inhomogeneous equations for $u_{j}$. As a solution of (3), we take the $N$-soliton solution [12] which includes the $N$ amplitude parameters $a_{j}$ and the $N$ phase parameters $\xi_{j}(j=1,2, \ldots, N)$. We note that in the limit of $t_{0} \rightarrow \infty, u_{0}$ is decomposed into a superposition of $N$ algebraic solitary waves, the $j$ th of which has $a_{j}$ and $\xi_{j}$ as the independent parameters. The fundamental assumption in the following discussion is that these parameters are slowly varying functions of $t_{j}(j \geq 1)$ in the presence of the perturbation. The time evolution of $a_{j}$ and $\xi_{j}$ is determined by the compatibility conditions such that Eq. (4) has bounded solutions. Explicitly, they can be expressed as

$$
\begin{array}{r}
\left(g_{j}, R\left[u_{0}\right]-u_{0, t_{1}}\right) \equiv \int_{-\infty}^{\infty} g_{j}\left(R\left[u_{0}\right]-u_{0, t_{1}}\right) d x=0 \\
(j=1,2, \ldots),
\end{array}
$$

where $g_{j}$ are solutions of the adjoint equation for the homogeneous part of (4), i.e.,

$$
g_{j, t_{0}}+4 u_{0} g_{j, x}+H g_{j, x x}=0 .
$$

The $2 N$ independent bounded solutions for (6) can be constructed as

$$
\begin{gathered}
g_{j}=\int_{-\infty}^{x} \frac{\partial u_{0}}{\partial a_{j}} d x \quad(j=1,2, \ldots, N), \\
g_{j+N}=\int_{-\infty}^{x} \frac{\partial u_{0}}{\partial \xi_{j}} d x \quad(j=1,2, \ldots, N) .
\end{gathered}
$$

An important observation at this stage is the following orthogonality relations which can be obtained with the aid of the $\mathrm{BO}$ equation (3):

$$
\begin{aligned}
&\left(g_{i}, \frac{\partial u_{0}}{\partial \xi_{j}}\right)=-\left(g_{i+N}, \frac{\partial u_{0}}{\partial a_{j}}\right)=\frac{\pi}{4} \delta_{i j} \\
&(i, j=1,2, \ldots, N), \\
&\left(g_{i}, \frac{\partial u_{0}}{\partial a_{j}}\right)=\left(g_{i+N}, \frac{\partial u_{0}}{\partial \xi_{j}}\right)=0 \\
&(i, j=1,2, \ldots, N),
\end{aligned}
$$

where $\delta_{i j}$ is Kronecker's delta. Since $u_{0}$ depends on $t_{1}$ through $a_{j}$ and $\xi_{j}, u_{0, t_{1}}=\sum_{j=1}^{N}\left(a_{j, t_{1}} u_{0, a_{j}}+\xi_{j, t_{1}} u_{0, \xi_{j}}\right)$. Substituting this relation into (5), using (9) and (10) and then rewriting the resultant equations in terms of the original time variable $t$, we finally obtain the time evolution of $a_{j}$ and $\xi_{j}$ as follows:

$$
\begin{gathered}
\frac{d a_{j}}{d t}=-\frac{4 \epsilon}{\pi}\left(g_{j+N}, R\left[u_{0}\right]\right) \quad(j=1,2, \ldots, N), \\
\frac{d \xi_{j}}{d t}=a_{j}-\frac{4 \epsilon}{\pi}\left(g_{j}, R\left[u_{0}\right]\right) \quad(j=1,2, \ldots, N) .
\end{gathered}
$$

It should be stressed that these results are valid over the time interval $0 \leq t \leq \epsilon^{-1}$. Beyond the upper limit of the interval, the higher-order modulation effects must be taken into account. The leading-order analysis here may be termed adiabatic approximation because it disregards the emission of radiation as well as the distortion of the shape of solitons due to perturbation. In order to elucidate these first-order effects, one must proceed to a higherorder approximation. To be more specific, it is necessary to solve Eq. (4) with $u_{0}$ being the $N$-soliton solution of Eq. (3). However, at present, I have no analytical means to resolve the problem. In this respect, it should be noted that the Green function method due to Keener and McLaughlin [17] seems to be inapplicable to the present first-order problem. One reason for this is that their method works well when the linear operator associated with the linearized equation [i.e., counterpart of the homogenous part of Eq. (4)] is self-adjoint with respect to appropriate inner product. Consequently, they could treat the perturbed nonlinear Schrödinger and sine-Gordon equations satisfactorily $[17,22]$ whereas the application to the perturbed $\mathrm{KdV}$ equation has been unsuccessful. In the case of the $\mathrm{KdV}$ equation with a small dissipation, the formation of a shelf in the lee of the solitary wave has been found in a first-order correction [15,23]. Whether similar phenomena occurs in the presence of dispersive perturbation is an important problem to be pursued in a future work. 
Let us now calculate the changes of the amplitude and the phase by employing (11) and (12). The corresponding changes of the $j$ th solitary wave may be represented by the relations

$$
\begin{aligned}
& \Delta a_{j}=\int_{-\infty}^{\infty}\left[\frac{d a_{j}}{d t}-\left(\frac{d a_{j}}{d t}\right)_{s_{j}}\right] d t \quad(j=1,2), \\
& \Delta \xi_{j}=\int_{-\infty}^{\infty}\left[\frac{d \xi_{j}}{d t}-\left(\frac{d \xi_{j}}{d t}\right)_{s_{j}}\right] d t \quad(j=1,2),
\end{aligned}
$$

where the subscript $s_{j}$ denotes the contribution from the $j$ th single solitary wave when there are no interactions. The above subtraction is necessary since we are seeking the net changes of the amplitude and the phase due to the interaction. In the present case, one finds that [11]

$$
\begin{gathered}
\left(\frac{d a_{j}}{d t}\right)_{s_{j}}=0 \quad(j=1,2) \\
\left(\frac{d \xi_{j}}{d t}\right)_{s_{j}}=a_{j}-\frac{3 \epsilon a_{j}^{2}}{16 \Delta^{2}}\left(31 \Delta^{2}+6\right) \quad(j=1,2) .
\end{gathered}
$$

We first calculate $\Delta a_{1}$ and $\Delta a_{2}$. It follows from the twosoliton solution of the BO equation [12] (7) and (8) that

$$
u_{0}=\frac{G}{F}, \quad g_{1}=\frac{G_{1}}{F}, \quad g_{3}=\frac{G_{3}}{F},
$$

with

$$
\begin{gathered}
F=\left[-\theta_{1} \theta_{2}+\left(\frac{s+1}{s-1}\right)^{2}\right]^{2}+\left(\theta_{1}+\theta_{2}\right)^{2}, \\
G=a_{1}\left[s \theta_{1}^{2}+\theta_{2}^{2}+\frac{(s+1)^{3}}{(s-1)^{2}}\right], \\
G_{1}=a_{1}^{-1}\left[\theta_{1} \theta_{2}^{2}-\frac{s+1}{(s-1)^{3}}\right. \\
\left.\times\left\{\left(-s^{2}+4 s+1\right) \theta_{1}+4 s \theta_{2}\right\}\right] \\
G_{3}=-a_{1}\left[\theta_{2}^{2}+\left(\frac{s+1}{s-1}\right)^{2}\right],
\end{gathered}
$$

where $\theta_{j}=a_{j}\left(x-\xi_{j}\right)\left(\xi_{j, t_{0}}=a_{j}\right)$ and $s=a_{2} / a_{1}(>1)$ is the amplitude ratio. In these expression, the amplitude and the phase of the larger (shorter) solitary wave are given respectively by $a_{2}\left(a_{1}\right)$ and $\xi_{2}\left(\xi_{1}\right)$. Substituting (15), (16), and (17)-(21) into (13) and (14), we immediately see that all the calculations reduce to the double integral of rational functions in $t$ and $x$. To carry out the integrals, it is convenient to introduce the new variables $z_{1}$ and $z_{2}$ by $z_{1}=\left(\theta_{1}+\theta_{2}\right) / 2$ and $z_{2}=\left(\theta_{1}-\theta_{2}\right) / 2$, so that the Jacobian of the transformation from $(t, z)$ to $\left(z_{1}, z_{2}\right)$ yields

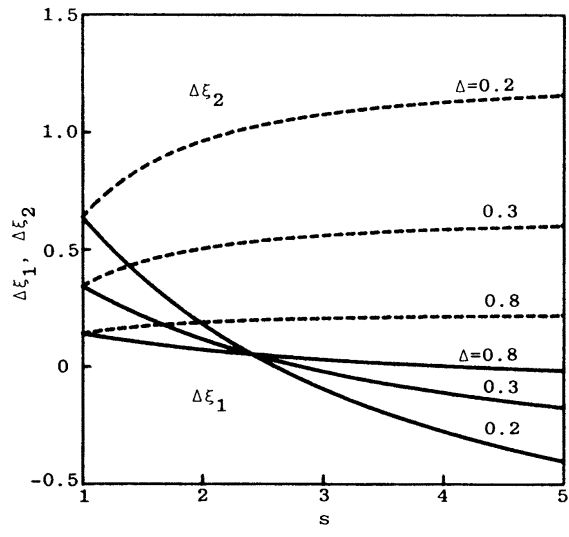

FIG. 1. Total phase shifts as a function of the amplitude ratio in the case of $\epsilon=0.003$ and $\Delta=0.2,0.3,0.8$. The solid and broken lines represent $\Delta \xi_{1}$ and $\Delta \xi_{2}$, respectively.

$2 /\left[a_{1}^{3} s(s-1)\right]$. As for $\Delta a_{1}$, one finds without any further calculation that $\Delta a_{1}=0$ since the integrand becomes the odd functions of $z_{1}$ or $z_{2}$. Similarly, one obtains $\Delta a_{2}=0$. Hence, up to the order $\epsilon$, the amplitudes of solitary waves do not change after overtaking collision between them.

On the other hand, the calculation of $\Delta \xi_{1}$ and $\Delta \xi_{2}$ is quite lengthy. It turns out that the integration with respect to $z_{1}$ is represented by a weighted sum of the associated Legendre functions $P_{j}^{-7 / 2}(\cos \theta)(j=0,1, \ldots, 4)$ with

$$
\cos \theta=2\left[z_{2}^{2}+\left(\frac{s+1}{s-1}\right)^{2}\right]^{-1}-1 .
$$

The integration in $z_{2}$ is then performed straightforwardly. All the algebraic calculations were done systematically with the help of the algebraic programming system REDUCE. The final result for $\Delta \xi_{1}$ is given as follows:

$$
\begin{aligned}
\Delta \xi_{1}=-\frac{3 \pi \epsilon}{4 \Delta^{2}(s+1)^{2}} & {\left[6\left(s^{2}-2 s-1\right)\right.} \\
& \left.+\left(8 s^{2}-46 s-23\right) \Delta^{2}\right] .
\end{aligned}
$$

By the same way, one obtains for $\Delta \xi_{2}$,

$$
\begin{aligned}
\Delta \xi_{2}=\frac{3 \pi \epsilon}{4 \Delta^{2}(s+1)^{2}} & {\left[6\left(s^{2}+2 s-1\right)\right.} \\
& \left.+\left(23 s^{2}+46 s-8\right) \Delta^{2}\right] .
\end{aligned}
$$

The total phase shifts $\Delta \xi_{1}$ and $\Delta \xi_{2}$ are plotted in Fig. 1 as a function of $s$ for several values of $\Delta$, where $\epsilon$ has been chosen to be 0.003 .

It is seen from (23) that the larger solitary wave always suffers a positive phase shift irrespective of values of $s$ and $\Delta$. The situation is quite different for the shorter solitary wave. Indeed, $\Delta \xi_{1}$ changes the sign according 


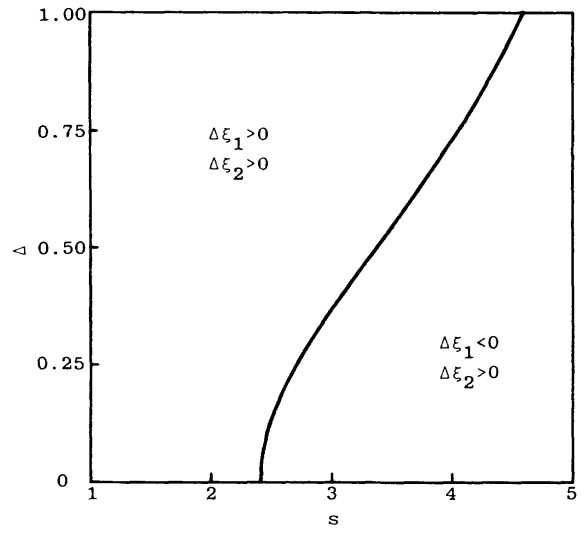

FIG. 2. Critical curve $\Delta$ vs $s$ that corresponds to $\Delta \xi_{1}=0$.

to values of $s$ and $\Delta$. There exists a critical curve $\Delta$ vs $s$ that corresponds to $\Delta \xi_{1}=0$ (see Fig. 2). In the left region surrounded by the curve, $\Delta \xi_{1}>0$ whereas in the right region, $\Delta \xi_{1}<0$. However, for values of $s$ in the range $s>4.594, \Delta \xi_{1}$ always takes a negative value. Also we can observe that the maximum deviations of $\Delta \xi_{j}(j=1,2)$ decrease as the value of $\Delta$ increases.

In conclusion, we have demonstrated analytically that the phase shifts occur after overtaking collision of two interfacial solitary waves. Although the algebraic solitary waves have been found in various model NEEs [12,24], they never exhibit the phase shift during the interaction process. Therefore, the result presented in this Letter is the first example of the phase shift that takes place between algebraic solitary waves. To confirm the above analytical prediction, one may perform direct numerical simulations on the basis of Eq. (2). In addition, it seems to be worthwhile to carry out an experiment analogous to that conducted for the purpose of determining the regions of applicability of various theories dealing with finiteamplitude interfacial solitary waves in a two-layer fluid system [25].

The author would like to thank Professor M. Nishioka for continual encouragement.

[1] G. B. Whitham, Linear and Nonlinear Waves (Wiley, New York, 1974).

[2] Waves on Fluid Interface, edited by R. E. Mayer (Academic, New York, 1983).

[3] Nonlinear Water Waves, edited by K. Horikawa and H. Maruo (Springer-Verlag, New York, 1988).
[4] C. H. Su and R. M. Mirie, J. Fluid Mech. 98, 509 (1980).

[5] R. M. Mirie and C. H. Su, J. Fluid Mech. 147, 213 (1984).

[6] J. G. B. Byatt-Smith, J. Fluid Mech. 182, 485 (1987); 197, 503 (1988); 205, 573 (1989).

[7] R. M. Mirie and C. H. Su, J. Fluid Mech. 115, 475 (1982).

[8] J.D. Fenton and M. M. Rienecker, J. Fluid Mech. 118, 411 (1982).

[9] M. Funakoshi and M. Oikawa, J. Phys. Soc. Jpn. 51, 1018 (1982).

[10] Q. Zou and C. H. Su, Phys. Fluids 29, 2113 (1986).

[11] Y. Matsuno, Phys. Rev. E 49, 2091 (1994).

[12] Y. Matsuno, J. Phys. A 12, 619 (1979).

[13] Y. Matsuno, J. Phys. A 13, 1519 (1980).

[14] Y. Matsuno, Bilinear Transformation Method (Academic, New York, 1984).

[15] D. J. Kaup SIAM J. Appl. Math. 31, 121 (1976); D. J. Kaup and A.C. Newell, Proc. R. Soc. London A 361, 413 (1978); V. I. Karpman and E. M. Maslov, Phys. Lett. 60A, 307 (1977); Sov. Phys. JETP 46, 281 (1977); 48, 252 (1978); V. I. Karpman, Phys. Scr. 20, 462, (1979).

[16] M. Tanaka, J. Phys. Soc. Jpn. 49, 807 (1980); K. A. Gorshkov and L. A. Ostrovsky, Physica (Amsterdam) 3D, 428 (1981); Y. Kodama and M. J. Ablowitz, Stud. Appl. Math. 64, 225 (1981); R. Grimshaw and H. Mitsudera, Stud. Appl. Math. 90, 75 (1993).

[17] J.P. Keener and D.W. McLaughlin, Phys. Rev. A 16, 777 (1977); R. L. Herman, J. Phys. A 23, 2327 (1990); L. A. Kalyakin, Theor. Math. Phys. 92, 736 (1992); V. V. Konotop and V.E. Vekslerchik, Phys. Rev. E 49, 2397 (1994).

[18] A. Bondeson, M. Lisak, and D. Anderson, Phys. Scr. 20, 479 (1979); J.-C. Fernandez, C. Froeschle, and G. Reinisch, Phys. Scr. 20, 545 (1979).

[19] M. Oikawa and N. Yajima, J. Phys. Soc. Jpn. 34, 1093 (1973); Prog. Theor. Phys. Suppl. 55, 36 (1974).

[20] Y. Kivshar and B. A. Malomed, Rev. Mod. Phys. 61, 763 (1989).

[21] Y. Matsuno (to be published).

[22] J.P. Keener and D. W. McLaughlin, J. Math Phys. 18, 2008 (1977).

[23] C. J. Knickerbocker and A. C. Newell, J. Fluid Mech. 98, 803 (1980).

[24] H. Airault, H.P. Mckean, and J. Moser, Commun. Pure Appl. Math. 30, 95 (1977); S. V. Manakov, V.E. Zakharov, L. A. Bordag, A.R. Its, and V.B. Matveev, Phys. Lett. 63A, 205 (1977); M. J. Ablowitz and J. Satsuma, J. Math. Phys. 19, 2180 (1978); J. Satsuma and M. J. Ablowitz, J. Math. Phys. 20, 1496 (1979); Y. Matsuno, J. Phys. Soc. Jpn. 57, 1577 (1989); J. Math. Phys. 31, 2904 (1990); C. P. Gilson and J. J. C. Nimmo, Phys. Lett. A 147, 472 (1990); .

[25] C. G. Koop and G. Butler, J. Fluid Mech. 112, 225 (1981). 